\title{
Development of a low-cost autofocusing probe for profile measurement
}

\author{
Kuang-Chao Fan ${ }^{1,3}$, Chih-Liang Chu ${ }^{1}$ and Jong-I Mou ${ }^{2}$ \\ ${ }^{1}$ Department of Mechanical Engineering, National Taiwan University, Taipei, Taiwan, \\ Republic of China \\ ${ }^{2}$ Department of Industrial Engineering, Arizona State University, Tempe, AZ, USA \\ E-mail: fan@ccms.ntu.edu.tw
}

Received 7 June 2001, in final form 30 August 2001, accepted for publication 27 September 2001

Published 6 November 2001

Online at stacks.iop.org/MST/12/2137

\begin{abstract}
In this research, a low-cost and precision autofocus laser probe system was developed. Modified from the pickup head of a commercially available DVD as a basis, it can detect the focus error signal (FES) of the object with a built-in four-quadrant photodiode. The FES will feed back to the developed controller, through which the objective lens can be adjusted in position automatically to remain in focus, driven by a voice coil motor (VCM). The driving current (converted from servo-FES) of the VCM and the objective lens displacement are one to one linearly related within several hundred micrometres. Because of this relationship, the surface profile and the surface roughness of the tested object can be realized. Calibrated results showed that the designed probe has $200 \mu \mathrm{m}$ linearity range, $0.2 \mu \mathrm{m}$ accuracy, $0.1 \mu \mathrm{m}$ resolution and standard deviation of $0.2 \mu \mathrm{m}$ on average. Some examples are given to show the applicability of this probe system.
\end{abstract}

Keywords: DVD pickup head, autofocus, voice coil motor, focus error signal

(Some figures in this article are in colour only in the electronic version; see www.iop.org)

\section{Introduction}

Technologies of ultra-precision manufacturing have rapidly grown in recent years. The corresponding inspection technologies with both the resolution and accuracy achieving submicrometre or even to nanometre level have also received great attention worldwide. From a precision perspective, micrometre probes are becoming less adequate, while finer measuring probes have high cost and sophistication. Hence the need for developing a new measuring probe to have high precision capability at a lower cost is well justified.

From past research $[1,2]$, the use of an optical probe enables a non-contact measuring approach. Using optical noncontact properties, precise measurements are made without damage to the measured object due to the absence of contacting force. In addition, as the spot size is very small, there is no need to compensate for the probe radius effect.

${ }^{3}$ Corresponding author.
A commercially available CD (compact disk) or DVD (digital versatile disk) pickup head contains the following components: laser diode, grating, polarization beam splitter and $1 / 4 \lambda$ plate, four-quadrant photodiode IC, objective lens and voice coil motor (VCM). Using the advanced packaging technology to combine these components results in a very useful tool for the purpose of optical measurement research.

Benschop and Rosmalen [3] replaced the laser diode in the CD pickup head by a high power laser through fibre optics as a new light resource to develop a scanning optical microscope. The four-quadrant photodiode and autofocus function were combined to sense the profile of the measured object surface. A confocal photodiode was attached near the laser source to sense the phase shift of the reflected light and thus scan the object surface condition.

Armstrong and Fitzgerald [4] developed a low-cost autocollimator based on the laser head of a $\mathrm{CD}$ player. The objective lens was removed to allow the reflected light to directly reach the four-quadrant photodiode. The voltage 
difference between the paired photodiodes was used to interpolate the angle changes in two perpendicular directions. The developed system has a measurement range of $1200 \mu \mathrm{rad}$ and a resolution of $\pm 0.006 \mu \mathrm{rad}$.

Quercioli et al [5] used the properties of the laser pickup head to develop a velocimeter. The laser beam passes through a grating, diffracts into three beams and finally reaches the measured disk surface. By knowing the distance between two light beams and calculating the delayed time between two reflected lights, the flying speed of the measured object can be determined.

Zhang and Cai [6] locked the voice coil motor in the pickup head and used a piezoelectric translator (PZT) to move the pickup head so as to make an autofocusing surface roughness measurement system possible. With the positioning accuracy of the PZT, the developed system has resolution to $10 \mathrm{~nm}$ and accuracy to $50 \mathrm{~nm}$. However, the PZT requires hundreds of volts as driving power and the measuring range is only about $30 \mu \mathrm{m}$, which limits its applications. Moreover, to replace the original installed VCM by PZT will change the structure and make the system more complicated.

In our previous study [7], the present authors also locked the voice coil motor in a CD pickup head and treated the head as a focusing probe. Using the one to one relationship between focus error magnitude and focus error signal (FES) in the S-curve domain, the height variation of a measured surface can be accurately measured. The developed system has the measurement accuracy of $34 \mathrm{~nm}$ and the measurement range of $10 \mu \mathrm{m}$.

In this report, the complete function of a DVD pickup head is fully utilized. The VCM can be activated by the developed servo-controller with built-in compensator circuit. A complete autofocusing probe can be realized. The measuring range can thus be enlarged to $200 \mu \mathrm{m}$ with calibrated accuracy to $0.2 \mu \mathrm{m}$.

\section{Probe system}

\subsection{Pickup head and autofocusing technique}

The pickup head uses a laser diode to produce optical light. Passing through a grating the light diffracts into three beams, as shown in figure 1 . These beams pass through a polarized beam splitter, a quarter wave plate and an objective lens and finally focus on the measuring surface. The beams are then reflected back along the original path and pass through a cylindrical lens, and finally project onto the four-quadrant photodiode, which will output a FES according to the main beam spot position in each quadrant. This focus error signal, after signal processing, is used to drive the VCM. The VCM shifts the objective lens until the focal point is back to the object surface. The amount of VCM movement is recorded and is equivalent to the profile change of the object.

This research employs the Sony DVD pickup head with model number KHM 220 [8]. Its focus theory is of the astigmatic method. As shown in figure 2, when the object plane is located inside or outside the focal plane of the objective lens the light on the four-quadrant photodetector will become an elliptical shape (plane 1, plane 3). If the object is right on the focus point, the light image will be in a circular shape (plane 2). Using proper signal processing, the focus error's

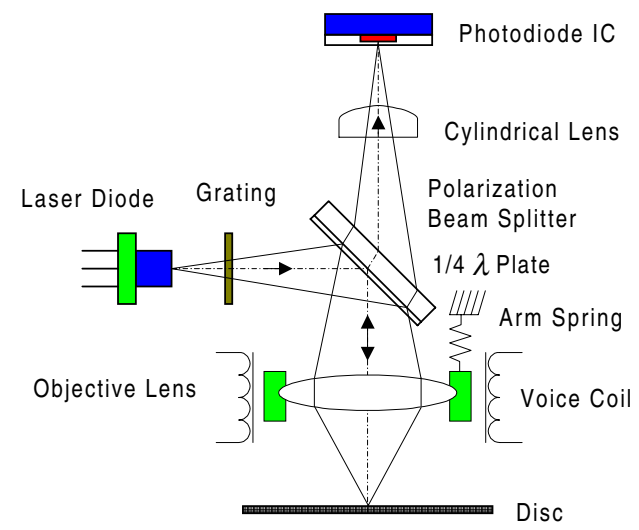

Figure 1. Structure of a DVD pickup head.

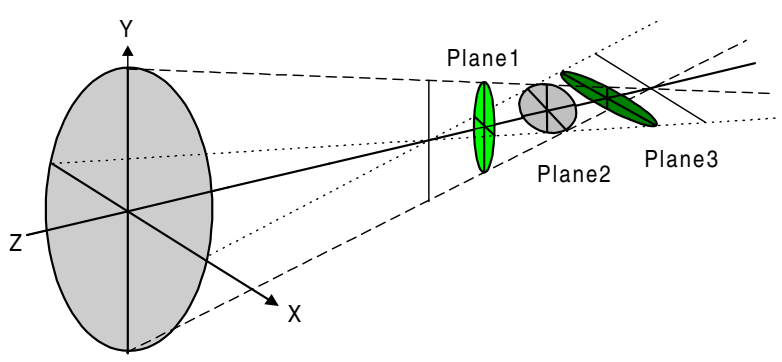

Figure 2. Schematic diagram of the astigmatic method.

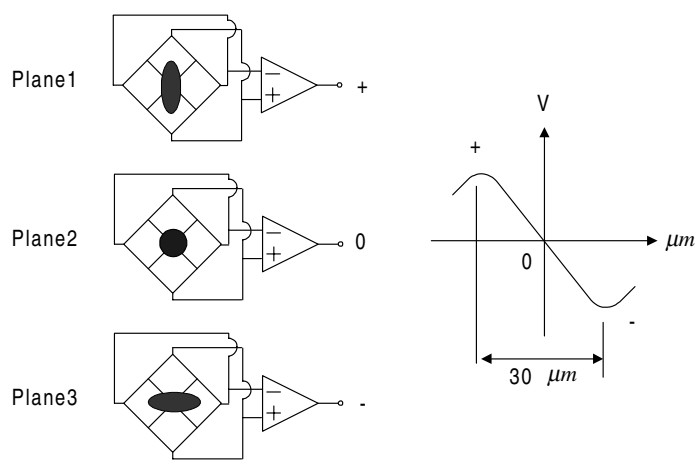

Figure 3. The variation of spot shapes with S-curve.

S-curve can be obtained by plotting the voltage difference between the paired photodiodes, as shown in figure 3 . The detectable range nearby the focal plane is about $30 \mu \mathrm{m}$ and the corresponding signal is called the focus error signal (FES) [7].

\subsection{Autofocusing theory}

As shown in figure 4, when the focus point is right on the reflecting surface the focus error signal will be zero. It corresponds to the middle position of the S-curve. When the object moves either closer to or away from the objective lens, the FES will have a non-zero output. This signal is processed and used to generate a current source to drive the VCM, which carries the objective lens so as to reduce the FES. Finally the lens stops its position when the mechanical system reaches the equilibrium condition and the FES returns to zero. The current needed to maintain the objective lens steadily will 


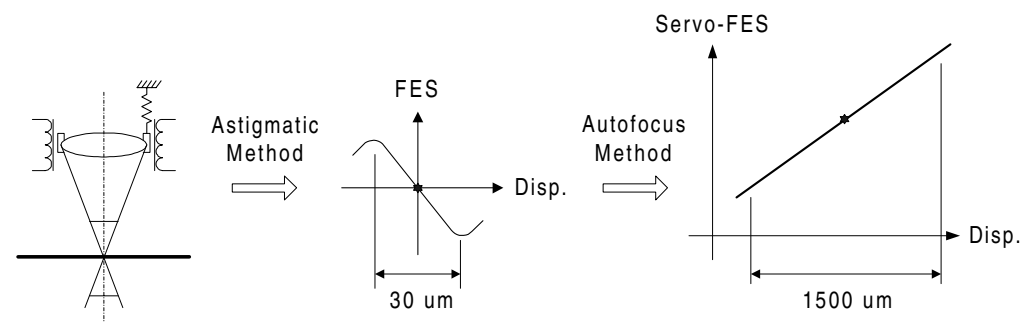

Figure 4. Servo-FES on the focal plane.

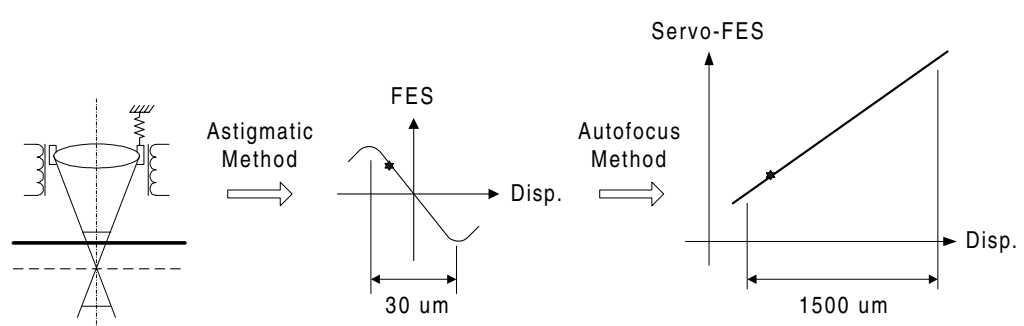

Figure 5. Servo-FES out of focal plane (too close).
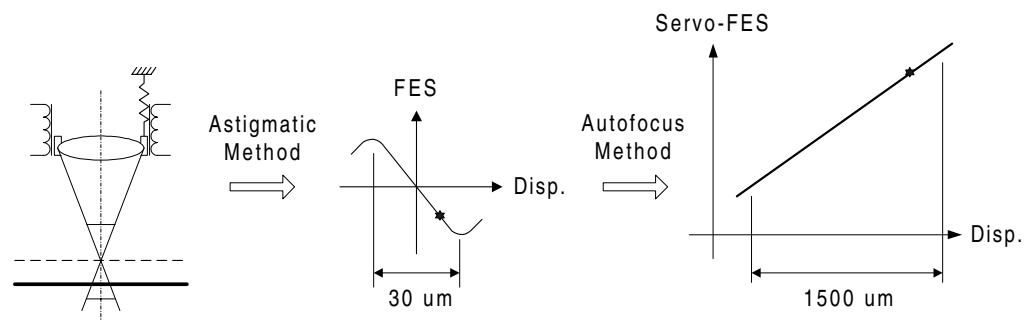

Figure 6. Servo-FES relationship far from focal plane.

be converted to a corresponding voltage signal, which is called the servo-FES, as shown in figures 5 and 6 . It is apparent that the autofocusing method can provide much larger linear range than the astigmatic (focusing) method. The amount of linear range can be varied according to different tested material and different laser power, which will be shown later in section 4 . The whole servo-controlled loop was designed with an analogue comparator and a built-in 8051 microprocessor for decision making in the present authors' laboratory. Since the balancing of the mechanical system, which will be analysed in the next session, is of real time phase, the observable results indicate the relationship between the lens displacement and the servo-FES in the steady-state condition.

In this study, the actual movement of the objective lens was calibrated by an HP 5529 laser interferometer. One example is given in figure 7, from which the linear range is found to be about $1700 \mu \mathrm{m}$. In order to achieve better linearity and accuracy, however, only the central range of approximately 200-300 $\mu \mathrm{m}$ (between 500 and $800 \mu \mathrm{m}$ ) was adopted in use.

\section{System integration and control}

\subsection{Mechanical system characteristics $[10,11]$}

In the laser pickup head, the mechanical system not only adjusts the objective lens as well as the VCM, it also provides elastic recovery force to balance with the magnetic force of

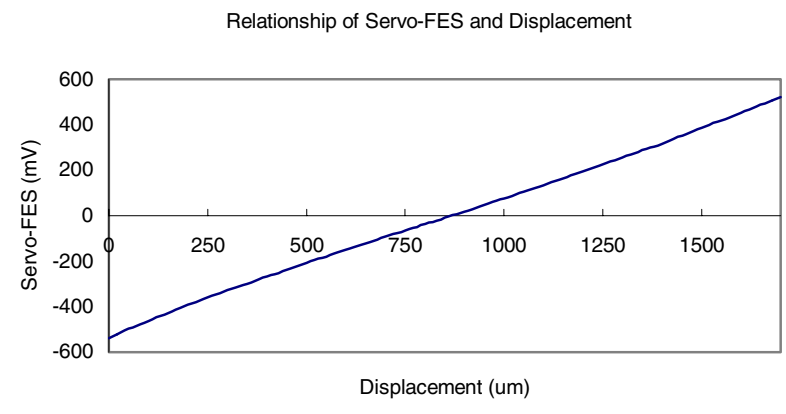

Figure 7. Relationship of the servo-FES and lens displacement.

the VCM. Such a system can be modelled with a second order forced vibration system, as shown in figure 8 . The moving part includes the VCM, the objective lens and the lens holder.

Using Laplace transformation the transfer function of the mechanical system can be expressed as follows

$$
\frac{X(s)}{F(s)}=\frac{1}{m s^{2}+c s+k}
$$

where $k$ denotes the spring constant and $c$ indicates the damping coefficient of the system. The closed loop transfer function can be expressed in the following form

$$
T(s)=\frac{C(s)}{R(s)}=\frac{\omega_{n}^{2}}{s^{2}+2 \xi \omega_{n} s+\omega_{n}^{2}}
$$




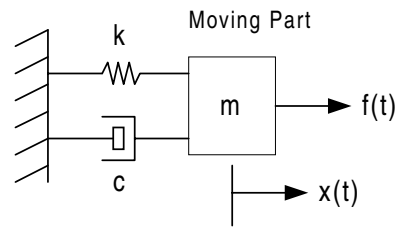

(a)

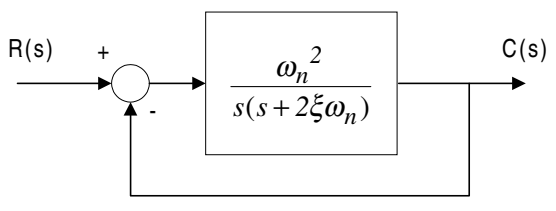

(b)

Figure 8. The lens/VCM system: (a) mechanical model, (b) second-order transfer function.

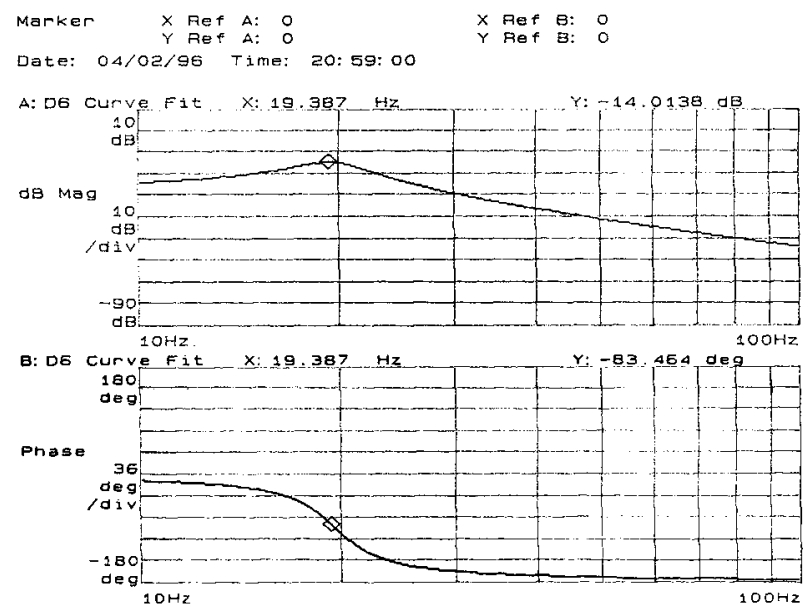

Figure 9. Spectrum of the lens/VCM system.

where $\omega_{n}$ is the natural frequency and $\xi$ is the damping ratio of the mechanical system.

An experiment was conducted with a vibration testing apparatus using a function generator to produce a sine wave current as input to the voice coil motor and using a laser fibre interferometer (Polytec model QFV502) to monitor the displacement of the objective lens. The system dynamic was analysed with an HP 4194A spectrum analyser. The dynamic characteristics of the mechanical system were found: the natural frequency is $19.4 \mathrm{~Hz}$ and the resonant peak is approximately $10 \mathrm{~dB}$ higher than the initial value, as shown in figure 9. As indicated in the service manual of the pickup head [8], the natural frequency is $20 \mathrm{~Hz}$, which is in good agreement with our experimental data. The moving body was weighed as $1.8 \mathrm{~g}$. From analysis [9], the system bandwidth was found to be $29.56 \mathrm{~Hz}$, the damping ratio is 0.161 , the damping coefficient $(c)$ is $0.07\left(\mathrm{~N} \mathrm{~S} \mathrm{~m}^{-1}\right)$, and the spring constant $(k)$ is $26.74\left(\mathrm{~N} \mathrm{~m}^{-1}\right)$. Therefore, equation (1) can be rewritten in the following form

$$
\frac{X(S)}{F(S)}=\frac{1}{0.0018 S^{2}+0.07 S+26.47}
$$

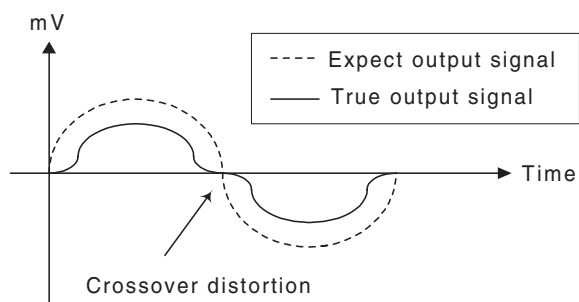

Figure 10. VCM crossover distortion.

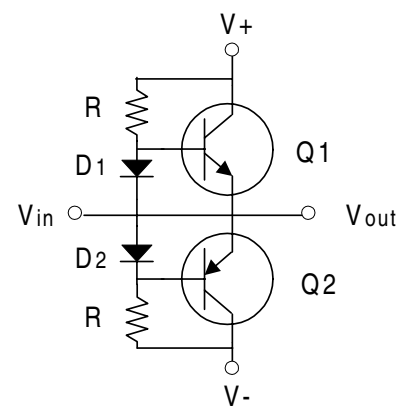

Figure 11. Bias-voltage compensated push-pull amplifier.

\subsection{Actuator system design and analysis}

In general, the output FES is very weak. Hence the FES must be amplified to a useable level to achieve the autofocusing purpose. It was found from experiments, however, if we just employed a simple push-pull amplifier [11], the VCM performed with crossover distortion, as shown in figure 10 . Through in-depth investigation it was found that the voltage difference between the base and emitter of the bipolar junction transistor used in the push-pull amplifier required at least $0.7 \mathrm{~V}$. Below this threshold level the signals would not be able to pass through the transistor. Therefore, a compensation circuit of a double push-pull circuit was developed to remove such a kind of distortion.

As shown in figure 11, two diodes $\left(D_{1}, D_{2}\right)$ were added in front of the original transistors $\left(\mathrm{Q}_{1}\right.$ and $\left.\mathrm{Q}_{2}\right)$ for the VCM pushpull circuit. The FES signal $\left(V_{i n}\right)$ was forced to pass through the $\mathrm{D}_{1}$ and $\mathrm{D}_{2}$ diodes first, thus causing the base voltage $V_{B}$ for positive transistor $\mathrm{Q}_{1}$ to have a larger voltage drop, $\mathrm{D}_{1}$, than the input voltage $V_{i n}$, and also causing the base voltage for negative transistor $\mathrm{Q}_{2}$ to have a larger voltage drop, $\mathrm{D}_{2}$, than the input voltage. Therefore, as the driving voltage for transistors $Q_{1}$ and $\mathrm{Q}_{2}$ is compensated, the crossover distortion can be eliminated. In this study, in order to assure the compatibility of the circuit, equivalent transistors $\left(\mathrm{Q}_{3}, \mathrm{Q}_{4}\right)$ to the diodes $\left(\mathrm{D}_{1}, \mathrm{D}_{2}\right)$ were selected. By selecting proper resistors, the double push-pull circuit can then be completed, as shown in figure 12 .

Using a function generator to produce a sine wave as the input for the double push-pull circuit and using an analogue scope to monitor the output signal, after crossover compensation the VCM motion can follow the input signal up to $10 \mathrm{kHz}$ without distortion, as shown in figure 13 .

\subsection{Autofocusing compensation control}

The autofocusing function of a pickup head is an integration of optical, electrical and mechanical systems. It can be 


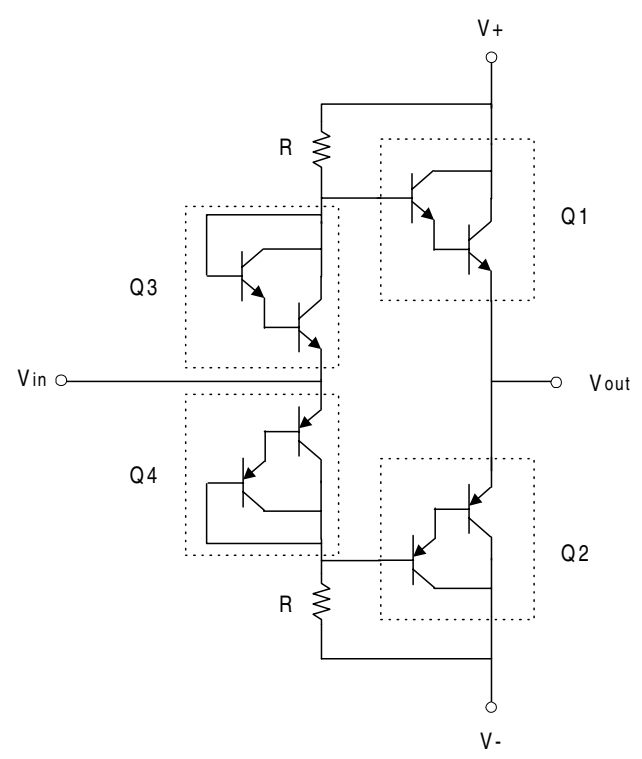

Figure 12. Developed double push-pull circuit for voice coil motor.

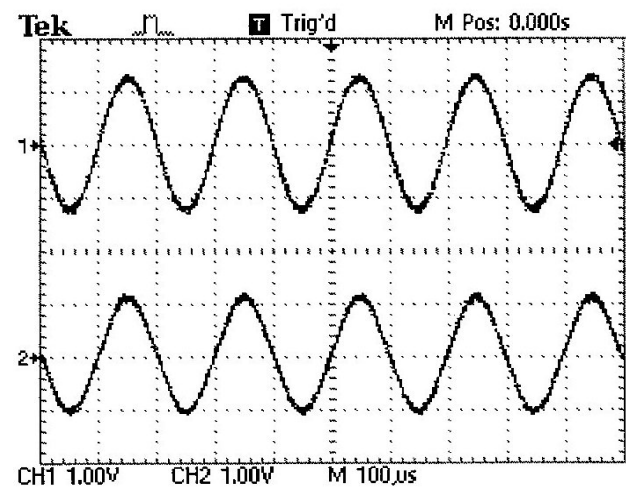

Figure 13. Crossover compensation results: (1) command input; (2) VCM response.

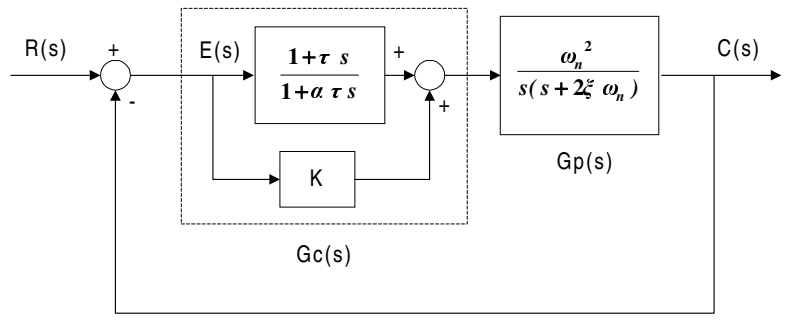

Figure 14. Compensation controlled block diagram.

found in figure 9 that the mechanical system has a significant response of the phase lag. Due to the difference in physical characteristics for each system, without proper control the system performance may be unstable and lead to degradation in measurement quality. A compensation controller was thus designed to increase the system gain and compensate for the phase lagging. As shown in figure $14, G_{c}(s)$ is a proportional and phase leading controller, which is called the compensation controller in this study, and $G_{p}(s)$ is the original mechanical system.
The transfer function of $G_{c}(s)$ loop can be derived in the frequency domain as follows

$$
G_{c}(\mathrm{j} \omega)=\frac{(1+K)+\mathrm{j} \omega(1+\alpha K) \tau}{1+\mathrm{j} \omega \alpha \tau}
$$

The gain and the phase can be determined as follows

$$
\begin{gathered}
\text { gain }=\left|G_{c}(\mathrm{j} \omega)\right|=\frac{\sqrt{(1+K)^{2}+(1+\alpha K)^{2} \tau^{2} \omega^{2}}}{\sqrt{1+\alpha^{2} \tau^{2} \omega^{2}}} \\
\text { phase }=\angle G_{c}(\mathrm{j} \omega)=\tan ^{-1}\left(\frac{(1+\alpha K) \tau \omega}{1+K}\right)-\tan ^{-1}(\alpha \tau \omega) .
\end{gathered}
$$

Obviously, the system's gain and phase values are influenced by $K, \alpha$ and $\tau$. For simplicity, $\tau$ is calculated from the $R C$ value in the circuit, $K$ is a variable and $\alpha=$ $\left(1-\sin \phi_{m}\right) /\left(1+\sin \phi_{m}\right)$ is still largely determined by $\phi_{m}$, the maximum phase lead. From the frequency response analysis [9], it was found that as the $K$ value increases the gain increases as well, while the corresponding phase lead decreases.

The compensation controller was implemented with an analogue circuit as shown in figure 15. Let $R_{6}=R_{7}=R_{8}=$ $R_{9}$; the frequency response function for the compensation controller circuit can be derived as follows

$$
H(\mathrm{j} \omega)=\frac{R_{5}}{R_{4}}+\frac{\mathrm{j} \omega R_{2} C_{1}}{1+\mathrm{j} \omega R_{1} C_{1}}+\frac{C_{2}}{C_{3}\left(1+\mathrm{j} \omega R_{3} C_{2}\right)} .
$$

Several experiments were conducted to investigate the best values of these three parameters, i.e. $K, \tau$ and $\phi_{m}$. Let $C_{1}=C_{2}=C_{3}=C$ and $R_{1}=R_{3}=R$, then substituting into equation (7) and comparing with equation (4) yields $K=R_{5} / R_{4}, \tau=R_{2}, \alpha=R / R_{2}$. Now, by setting the operational frequency as $10 \mathrm{~Hz}$ and the $\tau$ value as 0.016 , the system performance will be influenced by the values of $K$ and $\phi_{m}$. Let $\phi_{m}=30^{\circ}$, then $\alpha=0.333$. By setting the $K$ value from $1 / 6$ to 6 and executing the autofocusing function, the reflective surface displacement and servo-FES were recorded and plotted as shown in figure 16 . The results show that the larger the $K$ value, the longer the range that can be obtained.

Similar experiments were conducted for $\phi_{m}=45^{\circ}$ and $\phi_{m}=60^{\circ}$. It was found that to reach a suitable gain and phase lead, $\phi_{m}=45^{\circ}$ was the best selection, which led to an $\alpha$ value of about $0.1716, K=2$ and $\tau=0.016$. Under such conditions, the compensation controller provides a $15^{\circ}$ phase lead and $10 \mathrm{~dB}$ gain near the operational bandwidth, which is suitable for the requirement of phase leading compensation. Also the system gain increased slowly, which met the system requirements in force balance and avoiding amplified noise. The whole system diagram is shown in figure 17.

\section{Servo-FES characteristics}

\subsection{Laser power effect}

Two types of laser power input will affect the output signal of the laser diode, namely the voltage drift, which will influence the stability of the beam intensity, and the input current, which will change the intensity of the beam. 


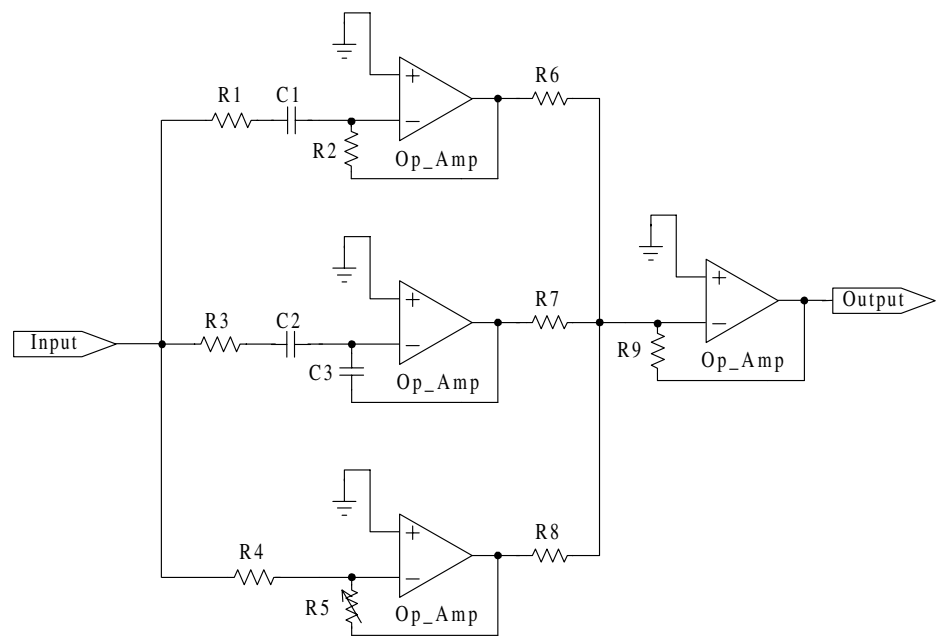

Figure 15. Developed compensation controller circuit.

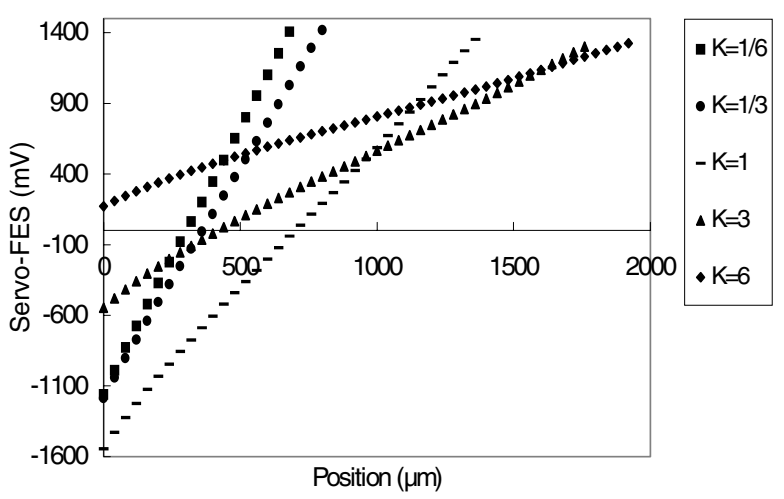

Figure 16. Reflective surface displacement and servo-FES relationship at $\phi_{m}=30^{\circ}$ and with various $K$ values.

For the developed measuring system shown in figure 1, the main reason for the laser diode's (LD's) focus drift and change of intensity power is because the LD output power is very sensitive to surrounding temperature. In this study, a laser power detector (PD) was embedded inside the pickup head and used to continuously monitor the LD's output power as feedback to the designed automatic power control (APC) circuit to control the stability of the laser output. The result shows (figure 18) that with an APC feedback circuit, the laser output power remains stable and does not decline as operation time increases. However, although the laser output power does not decline as operation time increases with APC feedback circuit, slight variation exists. Meanwhile, as shown in figure 19, various LD currents will lead to a change in servoFES slope. The slope drifts $0.09 \mu \mathrm{m} \mathrm{mV}^{-1}$ between 48.5 and $51.5 \mathrm{~mA}$. To ensure a precise measuring performance, a realtime servo-FES slope compensation technique was developed.

The LD operational current of the pickup head used in this study is about $52 \mathrm{~mA}$. To study the effect of the laser power effect on the autofocusing phenomenon, four LD current values were used to experimentally obtain the relationship between the servo-FES and the reflective surface displacement as shown in figure 20. The results show that with the larger laser driving current the displacement of the voice coil can be longer. Therefore, controlling and maintaining higher LD current supply will lead to a longer and effective autofocusing range with stable linearity.

\subsection{Reflection coefficient effect}

Different reflective surfaces will have various laser light absorption coefficients and yield the change of reflectivity. As shown in figure 21, the resulting S-curve will be different as well. Four different materials were used for autofocusing test and the results are shown in figure 22. Although the slopes of various S-curves are quite different, various autofocusing tests yield similar patterns of servo-FES. The major differences are revealed in the autofocusing ranges. A mercury $(\mathrm{Hg})$ mirror has the largest autofocusing range of $1300 \mu \mathrm{m}$. The $\mathrm{CD}$ reflective surface has slightly less autofocusing range than the mercury mirror, though its S-curve has a steeper slope. Meanwhile, though the S-curve of the aluminium film (Al) mirror has similar slope to that of the mercury mirror, its autofocusing range is surprisingly shorter. The autofocusing range of silicon wafer, that has the lowest reflection rate, is shortest as expected. Therefore, this study has demonstrated that the reflected light characteristics also have certain degrees of influence on the autofocusing and S-curve ranges.

\subsection{Analysis of the system's accuracy, repeatability and resolution}

Previous experimental results show that the relationship between the servo-FES and the objective lens displacement can be accurately determined as the laser power and compensation controller parameters are properly set. The system performance was calibrated by using an HP 5529 laser interferometer. The autofocusing function was repeatedly applied on a mercury mirror nine times. The servo-FES over a linear range between $600 \mu \mathrm{m}$ and $800 \mu \mathrm{m}$ was plotted in figure 23 and the slope was calculated and is tabulated in table 1. The averaged slope is $1.249 \mathrm{mV} \mu \mathrm{m}^{-1}$ and the averaged standard deviation is $0.255 \mathrm{mV}$. In terms of length, the standard deviation can be expressed by $0.2 \mu \mathrm{m}$ $\left(0.255 \mathrm{mV} /\left(1.249 \mathrm{mV} \mu \mathrm{m}^{-1}\right)\right)$. The measurement error, 


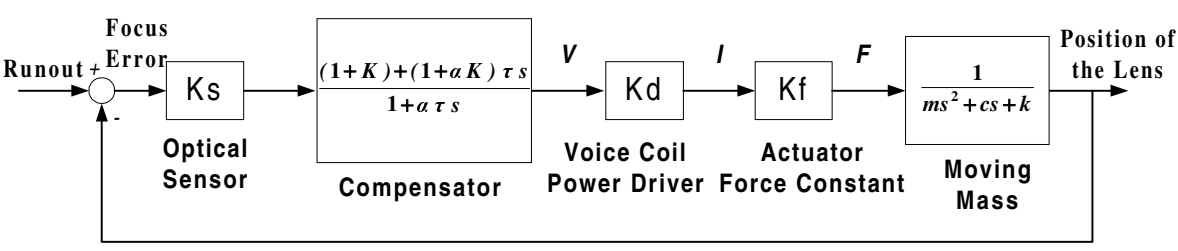

Figure 17. Servo-controlled system block diagram of the pickup head.

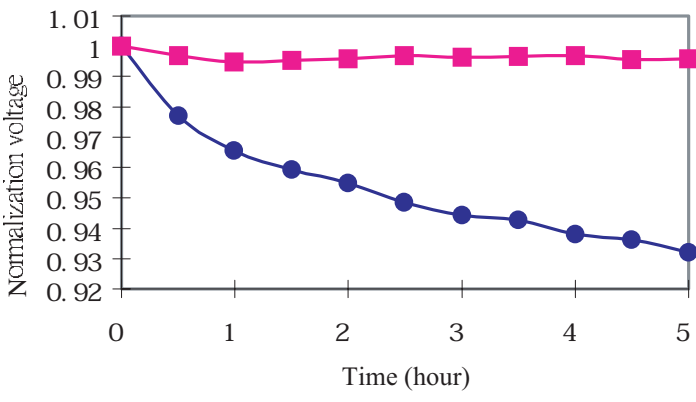

Figure 18. Laser output power against operation time.

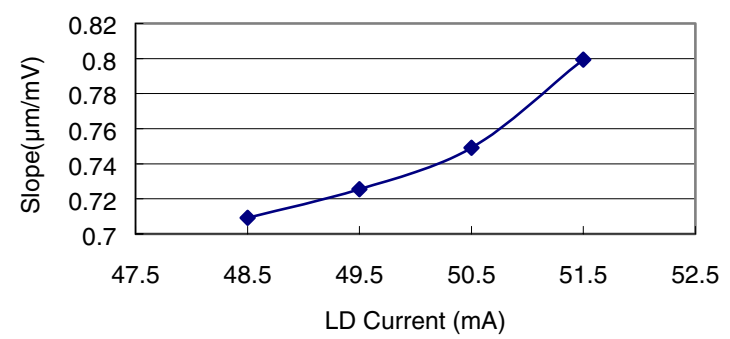

Figure 19. Effect of different LD currents on servo-FES slope.

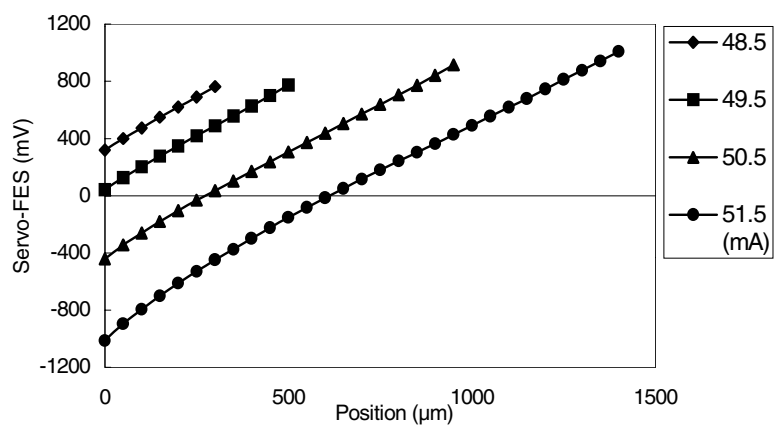

Figure 20. The effect of various LD currents on servo-FES.

determined as the product of averaged standard deviation and slope inverse, is $0.2 \mu \mathrm{m}$. This system employed a 12-bit $5 \mathrm{kHz}$ A/D converter of $\pm 5 \mathrm{~V}$ operational voltage to interface to a personal computer. One digit of the A/D corresponds to $2.44 \mathrm{mV}$. As calculated in table 1, the inverse of the autofocusing slope is $0.8 \mu \mathrm{m} \mathrm{mV} V^{-1}$. At each measuring point, the data were taken 20 times and averaged in realtime phase (about $4 \mathrm{~ms}$ ). The displayed resolution could thus reach $2.44(\mathrm{mV}) \times 0.8\left(\mu \mathrm{m} \mathrm{mV}^{-1}\right) / 20=0.096 \mu \mathrm{m}$. Thus, the system can be confirmed with the measured range of $200 \mu \mathrm{m}$, accuracy of $0.2 \mu \mathrm{m}$, resolution of $0.1 \mu \mathrm{m}$ and standard deviation of $0.2 \mu \mathrm{m}$ on average.

\section{Profile measurement applications}

Figure 24 plots the set-up and its system diagram of the autofocusing probe for profile measurement. The object was fixed and the pickup head was driven by a micropositioning stage so that the laser beam can scan across the tested surface. The stage, made by Parker (model number 106004BTMP), was initially calibrated in positioning accuracy with an HP 5529 laser interferometer and compensated for its errors to $0.1 \mu \mathrm{m}[7,12]$.

\subsection{Surface profile measurement}

The average track width for most compact disks is about $2 \mu \mathrm{m}$. With a proper control to move the micro-stage in $0.25 \mu \mathrm{m}$ incremental steps, the surface profile of a compact disk with $1.5 \mu \mathrm{m}$ track width and $0.2 \mu \mathrm{m}$ depth difference was able to be measured as shown in figure 25 .

The results show that the average measured track width was found to be about $1.534 \mu \mathrm{m}$, which is very close to the nominal value of $1.5 \mu \mathrm{m}$. The measured peak-to-valley depth ranges between 0.19 and $0.27 \mu \mathrm{m}$ with an average of $0.23 \mu \mathrm{m}$, which is slightly different from the nominal value of $0.2 \mu \mathrm{m}$. The errors could be due to the uneven etched depth of the CD and the spot size of the laser beam $(1 \mu \mathrm{m})$. Instead of an ideal point measurement this process can only achieve a small area measurement. The reflected signal from the CD surface is the averaged signal of the projected area. A slight variation in measured result cannot be avoided [7].

\subsection{Step height measurement}

Two grade-one gauge blocks with nominal lengths of $1.08 \mathrm{~mm}$ and $1.05 \mathrm{~mm}$ respectively were put together on a reference plane, as shown in figure 26 . The autofocusing probe scanned the surface across the step height. Received signals were dramatically influenced by the chamfer of each block, which reflected the light away from the sensor. The sum of light intensity accordingly dropped and hence the focus error signal (FES) jumped up [7]. To avoid this noised phenomenon, therefore, in this study two plane mirrors with the same thickness were placed on the top of the gauge blocks, and the measured results are shown in figure 27. The negative sign arises from multiplying the servo-FES with slope directly. The noiselike signal could be due to the straightness error of the stage. Since the moving axis of the micro-positioning stage and the gauge block surfaces would have misalignment error, slope of the measured data can be expected. The non-parallelism of the upper and lower lines arises from the imperfect mirror mounting. The least squares method was applied to determine the best fitted lines for each measured surface, as shown in 


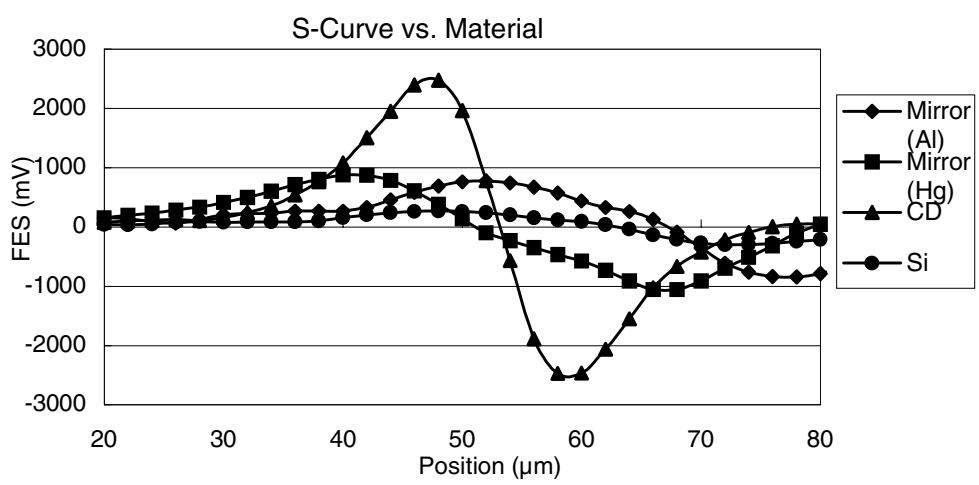

Figure 21. S-curves for various materials.

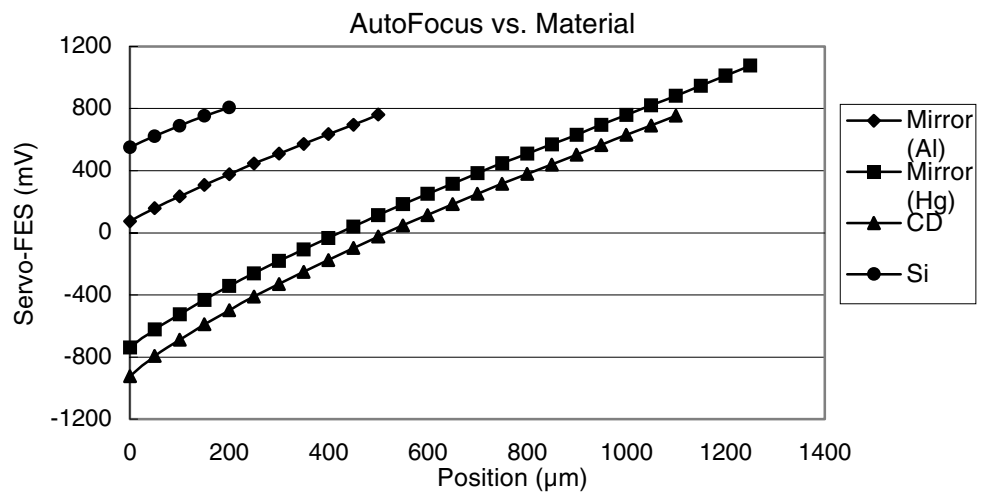

Figure 22. Autofocusing characteristics for various materials.

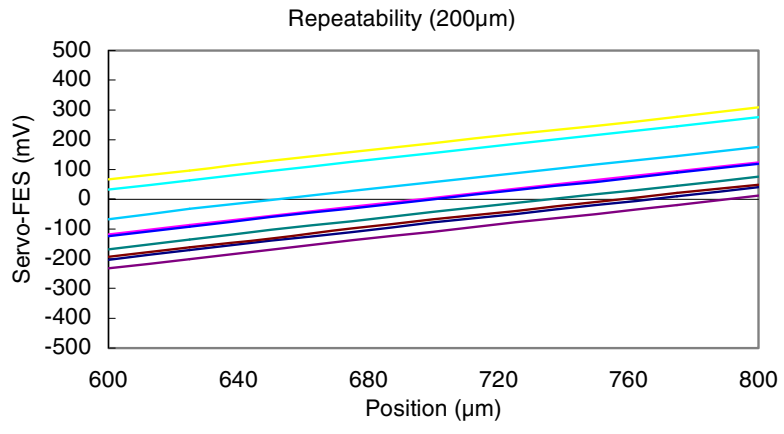

Figure 23. Repeatability analysis within a $200 \mu \mathrm{m}$ linear range.

figure 28. The step height of these two straight lines was found to be about $28.9 \mu \mathrm{m}$ at $X=1600$. Comparing with the nominal value, the measurement error of about 0.37 surface effect.

\subsection{Thickness measurement of thin film}

A silicon substrate was coated with a thin chromium film by the physical vapour deposition (PVD) process. The nominal film thickness was $2 \mu \mathrm{m}$. A small portion of the coated film was scratched off to expose the silicon substrate. By measuring the surface profile changes at the film-substrate interface this could lead the determination of film thickness. Since the coated film and the substrate are made of different materials, which would respond with different linearity curves, therefore recording the VCM displacements at two different focal points
Table 1. Calibration results.

\begin{tabular}{lllll}
\hline & $\begin{array}{l}\text { Standard } \\
\text { deviation } \\
(\mathrm{mV})\end{array}$ & $\begin{array}{l}\text { Slope } \\
\left(\mathrm{mV} \mu \mathrm{m}^{-1}\right)\end{array}$ & $\begin{array}{l}\text { Slope } \\
\text { inverse } \\
\left(\mu \mathrm{m} \mathrm{mV}^{-1}\right)\end{array}$ & $\begin{array}{l}\text { Error } \\
(\mu \mathrm{m})\end{array}$ \\
\hline 1 & 0.177 & 1.261 & 0.794 & 0.141 \\
2 & 0.269 & 1.262 & 0.793 & 0.214 \\
3 & 0.242 & 1.235 & 0.810 & 0.196 \\
4 & 0.306 & 1.240 & 0.806 & 0.246 \\
5 & 0.264 & 1.280 & 0.781 & 0.206 \\
6 & 0.330 & 1.252 & 0.799 & 0.264 \\
7 & 0.235 & 1.233 & 0.811 & 0.190 \\
8 & 0.231 & 1.241 & 0.806 & 0.186 \\
9 & 0.244 & 1.239 & 0.807 & 0.197 \\
Average & 0.255 & 1.249 & 0.801 & 0.205 \\
\hline
\end{tabular}

with respect to different material surfaces is more appropriate in this study. Figure 29 shows the measured profile across the coating edge. The spiking signals reflect the potential burrs at the scratched interface and the noised signals reflect the roughness of the coated surface. With the least squares method, we could fit a straight line for each of the tested material surfaces, as shown in figure 30. It is noted from Figure 22 that although different materials will have different linear ranges the slopes of the characteristic lines, or the sensitivities, are almost the same. This is why the slope coefficients of the two fitted lines are very close to each other. In this sense, the distance of these two lines could be readily found to be about $1.962 \mu \mathrm{m}$, which is quite close to the nominal value of $2 \mu \mathrm{m}$. 


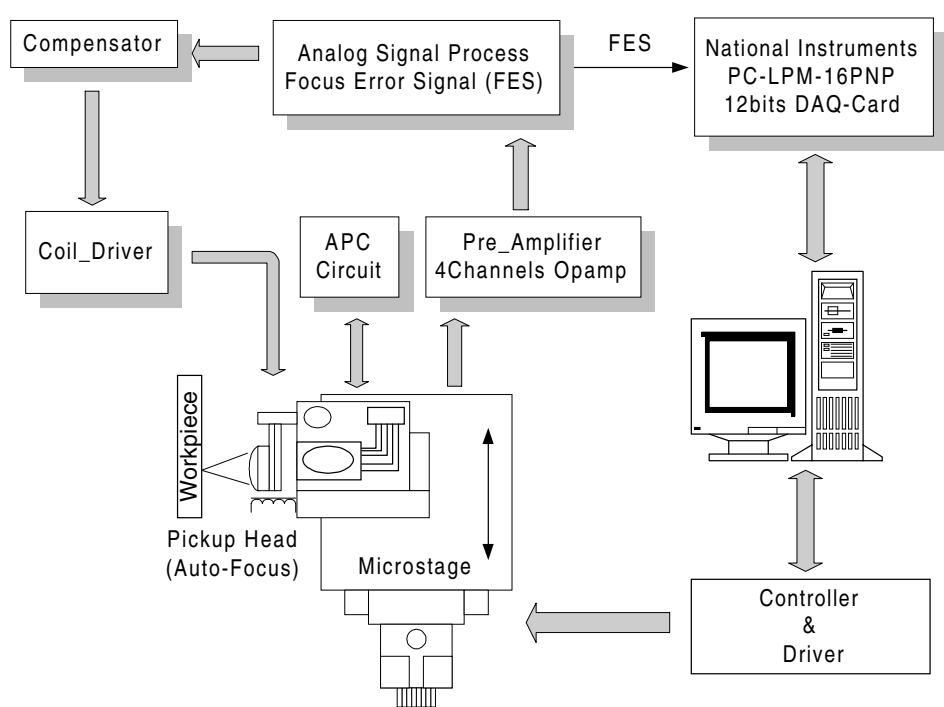

Figure 24. System setup for profile measurement.

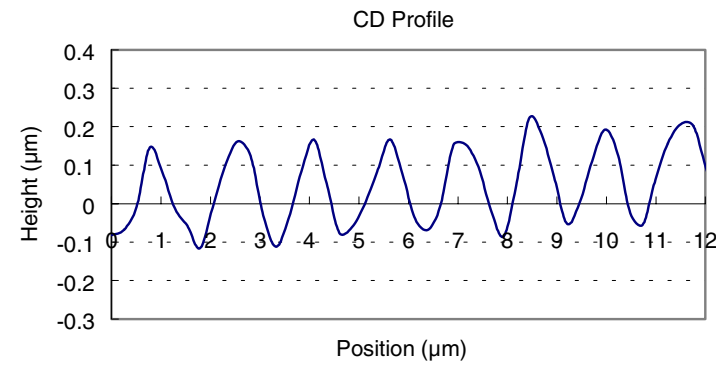

Figure 25. Measured profile of a compact disk.

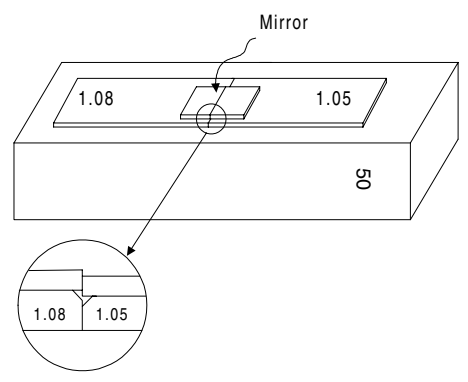

Figure 26. Setup for a step-height measurement.

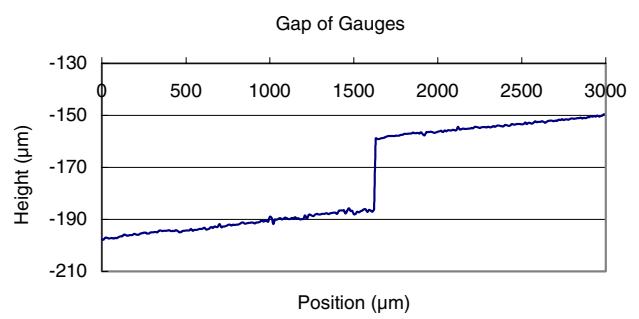

Figure 27. Step-height measurement results.

\section{Conclusions and discussion}

With today's advanced manufacturing technology, market demands continuously increase product precision and reduce

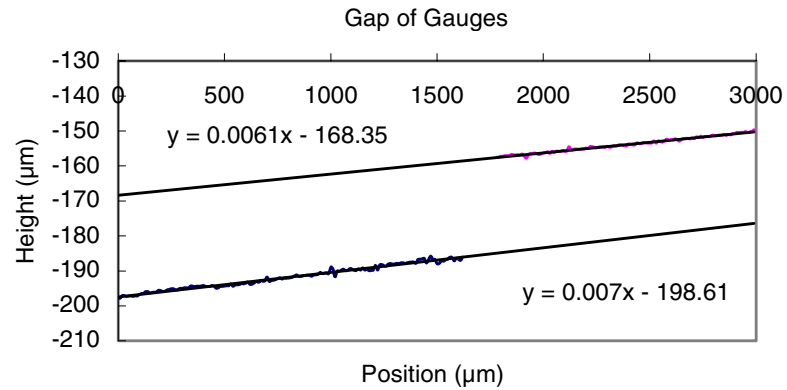

Figure 28. Extension of two measured lines to obtain the distance.

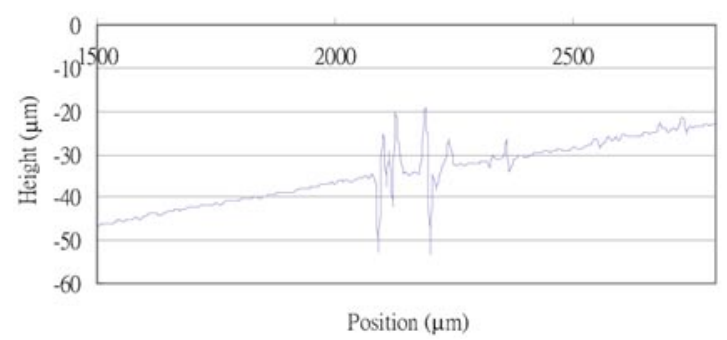

Figure 29. Measured thin film profile.

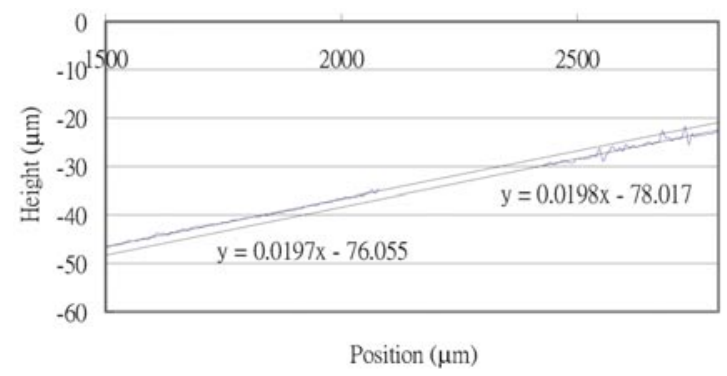

Figure 30. Extension of two lines after least squares fitting.

in physical size. To satisfy high precision measurement at non-destructive conditions, optical measuring probes are 
very useful. In this research, a DVD pickup head has been successfully modified to become a low-cost, high-precision and autofocusing measuring system. This system has a measuring range up to $200 \mu \mathrm{m}$ with accuracy about $0.2 \mu \mathrm{m}$, a resolution of $0.1 \mu \mathrm{m}$ and standard deviation of $0.2 \mu \mathrm{m}$ on average. The hardware cost of the developed system is about US\$100, which is significantly less than most commercial products with similar function and capability.

From this study it was also found that the VCM has a significant hysteresis effect. Because of its complexity in the compensator design this report does not discuss the methodology that has been developed. In example 1, the capability of measuring the up and down profile is simply because the range is within the focal range and using the S-curve signals can detect the profile variation straightforwardly. In the other two examples, only one directional motion of the lens can detect the step height of one material larger than the focal length, and the thin film thickness of the chromium on the glass substrate. Our results on compensation of the hysteresis effect will be published in the future.

\section{References}

[1] Whitehouse D J 1988 Comparison between stylus and optical methods for measuring surfaces Ann. CIRP 37 649-53
[2] Shiou Fang-Jung 1998 Entwicklung von Strategien und Messverfahren zur Erfassung dimensioneller Bauteilgroessen fuer die Laserbearbeitung Dissertation RWTH Aachen

[3] Benschop J and Rosmalen G V 1991 Confocal compact scanning optical microscope based on compact disc technology Appl. Opt. 30 1179-84

[4] Armstrong T R and Fitzgerald M P 1992 An autocollimator based on the laser head of a compact disc player Meas. Sci. Technol. 3 1072-6

[5] Quercioli F, Mannoni A and Tiribilli B 1997 Correlation optical velocimetry with a compact disk pickup Appl. Opt. 36 6372-5

[6] Zhang J H and Cai L L 1997 An autofocusing measurement system with a piezoelectric translator IEEE/ASME Trans. Mechatron. 2 213-16

[7] Fan K C, Lin C Y and Shyu L H 2000 Development of a low-cost focusing probe for profile measurement Meas. Sci. Technol. 11 1-7

[8] Sony 1995 Model Specifications KSM-213BCM

[9] Lin W J 1999 Development of an autofocusing probe system from the DVD pickup head Master Thesis National Taiwan University

[10] Bernacki B E and Mansuripur M 1994 Causes of focus-error feedthrough in optical-disk systems: astigmatic and obscuration methods Appl. Opt. 33 735-42

[11] Mansuripur M 1987 Analysis of astigmatic focusing and push-pull tracking error signals in magnet optical disk systems Appl. Opt. 26 3981-6

[12] Fan K C, Lin C Y and Shyu L H 1998 Positional error compensation of a precision microstage J. CSME $19543-8$ 